\title{
Central Nervous System Undifferentiated Pleomorphic Sarcoma
}

National Cancer Institute

\section{Source}

National Cancer Institute. Central Nervous System Undifferentiated Pleomorphic

Sarcoma. NCl Thesaurus. Code C129566.

A rare undifferentiated pleomorphic sarcoma (formerly known as malignant fibrous histiocytoma) involving the central nervous system. 